Gut and Liver, Vol. 12, No. 5, September 2018, pp. 523-529

\title{
Clinical Implication and Risk Factors for Malignancy of Atypical Gastric Gland during Forceps Biopsy
}

\author{
Min Seong Kim ${ }^{1}$, Sang Gyun Kim ${ }^{1}$, Hyunsoo Chung ${ }^{1}$, Jung Kim ${ }^{1}$, Hyoungju Hong ${ }^{1}$, Hee Jong Lee ${ }^{1}$, Hyun Ju Kim ${ }^{1}$,
} Min A Kim², Woo Ho Kim², and Hyun Chae Jung ${ }^{1}$

${ }^{\prime}$ Department of Internal Medicine and Liver Research Institute and ${ }^{2}$ Department of Pathology, Seoul National University College of Medicine, Seoul, Korea

Background/Aims: Although forceps biopsy is performed for suspicious gastric tumors during endoscopy, it is difficult to determine treatment strategies for atypical gastric glands due to uncertainty of the diagnosis. The aim of this study was to investigate clinical implications and risk factors for predicting malignancy in atypical gastric glands during forceps biopsy. Methods: We retrospectively reviewed medical records of 252 patients with a diagnosis of atypical gastric gland during forceps biopsy. Predictors of malignancy were analyzed using initial endoscopic findings and clinical data. Results: The final diagnosis for 252 consecutive patients was gastric cancer in 189 (75\%), adenoma in 26 (10.3\%), and gastritis in $37(14.7 \%)$. In the multivariate analysis, lesion sizes of more than $10 \mathrm{~mm}$ (odds ratio [OR], 3.021; 95\% confidence interval $[\mathrm{Cl}$ ], 1.480 to 6.165; $\mathrm{p}=0.002$ ), depressed morphology (OR, 3.181; 95\% Cl, 1.579 to 6.406, $p=0.001$ ), and surface nodularity (OR, 3.432; 95\% Cl, 1.667 to $7.064, p=0.001$ ) were significant risk factors for malignancy. Conclusions: Further evaluation and treatment should be considered for atypical gastric gland during forceps biopsy if there is a largesized (>10 mm) lesion, depressed morphology, or surface nodularity. (Gut Liver 2018;12:523-529)

Key Words: Atypical gland; Forcep biopsy; Stomach neoplasms; Adenoma; Gastritis

\section{INTRODUCTION}

Gastric cancer is the most common gastrointestinal malignancy in East Asia. ${ }^{1}$ The National Cancer Screening Program for gastric cancer in Korea has been conducted for adults over 40 years of age due to high prevalence of gastric cancer in this population. ${ }^{2}$ With increasing screening endoscopy, early detection of gastric cancer and precancerous lesions has also increased. Pathologic results of forceps biopsy for suspicious malignant lesions can be interpreted as atypical glands that are indeterminate results between malignancy and benign disease. Atypical gland is usually diagnosed when it is difficult to interpret between epithelial neoplasia and inflammatory change with histological morphology of abnormal epithelium and gland formation. ${ }^{3}$ Moreover, while endoscopic forceps biopsy is an initial favorable modality for the diagnosis of gastric tumor, the tissue may not be sufficient for definite diagnosis. ${ }^{4,5}$

Previous studies have attempted to determine clinical factors suggesting cancer for pathologic results of indefinite neoplasia in Vienna classification. ${ }^{6-8}$ Indefinite neoplasia could be further subcategorized into atypical gland/cellular atypia and reactive/ regenerative atypia. Atypical gland usually maintains a glandular structure to some extent that is more likely to be dysplasia while regenerative atypism/atypia is more likely to be an inflammatory change rather than tumor, though there is no clear boundary between them. ${ }^{9}$

Endoscopic re-biopsy is usually recommended according to the guideline if the pathology of initial biopsy results is atypical gland. ${ }^{10,11}$ However, if endoscopic findings are strongly suspected to be gastric cancer or high-grade adenoma, endoscopic resection may be a better option for definitive diagnosis and treatment than repeated biopsy. ${ }^{12}$ Recently, endoscopic submucosal dissection (ESD) has enabled definitive diagnosis and treatment by en-bloc resection of suspicious gastric tumor. It has replaced surgical treatment of early gastric cancer (EGC) in indicated cases and gastric adenoma as precancerous lesion. ${ }^{13,14}$

At present, there have been no studies merely analyzing clinical features of "atypical gland" excluding "regenerative atypia."

Correspondence to: Sang Gyun Kim

Department of Internal Medicine and Liver Research Institute, Seoul National University College of Medicine, 101 Daehak-ro, Jongno-gu, Seoul 03080, Korea

Tel: +82-2-740-8112, Fax: +82-2-743-6701, E-mail: harley1333@hanmail.net Received on January 2, 2018. Revised on April 8, 2018. Accepted on April 19, 2018. pISSN 1976-2283 eISSN 2005-1212 https://doi.org/10.5009/gnl18006

@ This is an Open Access article distributed under the terms of the Creative Commons Attribution Non-Commercial License (http://creativecommons.org/licenses/by-nc/4.0) which permits unrestricted non-commercial use, distribution, and reproduction in any medium, provided the original work is properly cited. 
The aim of this study was to determine the clinical implication of atypical gland at initial forceps biopsy and factors for predicting gastric cancer in endoscopic findings.

\section{MATERIALS AND METHODS}

\section{Patients}

Between March 2006 and February 2016, patients who were pathologically diagnosed as atypical gland at forceps biopsy for suspicious gastric tumor at Seoul National University Hospital were enrolled in this study. For patients who were referred from other clinics with diagnosis of atypical gland in pathology, the diagnosis was confirmed by pathologic review of the slide.

Atypical gland was considered when there were pathologic characteristics of dysplasia (cellular atypia, abnormal differentiation, disorganized mucosal architecture) with too good differentiation; Dysplasia appeared in extremely fine part of the regenerative atypia was excluded because of its pathologic features of benign lesion like gastritis with distribution of surrounding inflammatory cells (Fig. 1). ${ }^{9}$

The present study was approved by the Institutional Review Board of Seoul National University Hospital (IRB No. H-1704124-848). It was conducted in accordance with the Declaration of Helsinki. The informed consent was waived with the retrospective nature.

\section{Clinical outcomes and histopathological evaluation}

Medical records of patients were collected and evaluated for basic clinical and endoscopic findings. Each endoscopic image and report were reviewed for the description of diameter and gross features of the lesion. Endoscopic photographs were taken using the following two versions of endoscope (GIF-H260/ H290; Olympus Optical Co., Ltd., Tokyo, Japan). Gross type (elevated, flat, depressed), surface redness, nodularity, presence of ulcer, location of the lesion, endoscopic presence of intestinal metaplasia in background mucosa, and status of Helicobacter pylori infection were collected. The status of $H$. pylori infection was classified into four stages (negative to 3 positive) according to the density of $H$. pylori based on the result of pathologic examination of the specimen. Surface redness was defined as a red discoloration on the mucosal surface. Nodularity was defined as the presence of irregular elevation or nodular mucosa. ${ }^{15}$ Location of the lesion was divided into three identical sections: upper, middle, and lower. ${ }^{16}$

For cases who had undergone ESD or surgical resection, serial sections with thickness of $2 \mathrm{~mm}$ were made in the case of ESD and $4 \mathrm{~mm}$ sections were made in case of surgical resection for histological mapping. Gastric cancer was classified according to World Health Organization (WHO) classification method and Japanese Gastroenterological classification according to the degree of differentiation. ${ }^{16,17}$

\section{Statistical analyses}

For comparison between malignant and benign diseases, we divided patients into two subgroups. Chi-square test, Fisher exact test, and Student t-test were used to analyze the relationship among variables suggestive malignancy with univariate analysis. Multiple logistic regression analyses were used to examine independent risk factors for multivariate analysis with a pvalue of less than 0.05 in univariate analysis. SPSS Statistics for Windows version 23.0 (IBM Corp., Armonk, NY, USA) was used for all statistical analyses. Null hypotheses of no difference were rejected if p-values were less than 0.05 .

\section{RESULTS}

\section{Baseline characteristics}

During the study period, 859 patients were diagnosed or referred from outside clinic as atypical gland, in which 587 patients were excluded due to coincidental adenoma/adenocarcinoma or no atypical gland at slide review. In addition, 20 patients were excluded because of follow-up loss or other diagno-
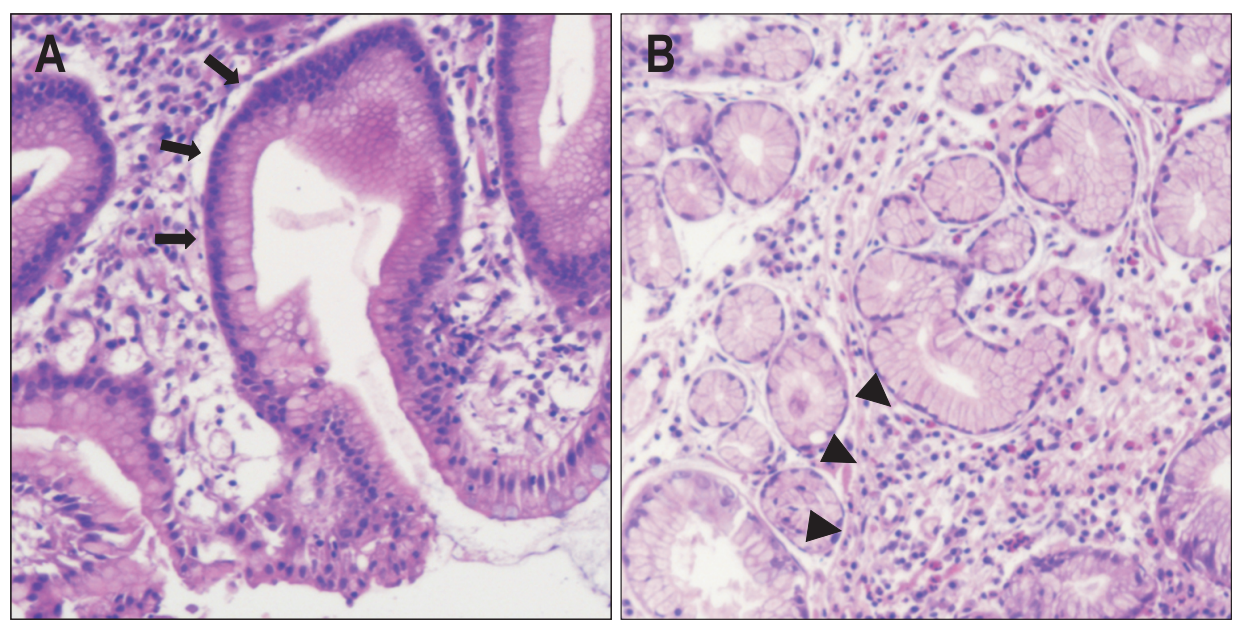

Fig. 1. Typical pathology photograph of an atypical gland and regenerative atypia in indefinite neoplasia. (A) Atypical gland similar to dysplasia with good differentiation (arrows). (B) Regenerative atypia with numerous inflammatory cells (triangles). 
sis (fibroelastoma, gastrointestinal stromal tumors). Finally, 252 patients were analyzed for the diagnosis of atypical gland (Fig 2). The malignant group consisted of 141 men and 48 women with a mean age of 61.5 years while the benign group consisted of 49 men and 14 women with a median age of 64.6 years. Of these, initial endoscopic resection or surgical resection was performed for definitive treatment in 48 and 23 patients, respectively. Additional endoscopic biopsy was performed in 181 patients, of which 32 patients remained to have the diagnosis of atypical gland at secondary biopsy, seven patients remained at tertiary biopsy, and two patients remained in the fourth biopsy (Fig. 3). Final diagnoses of consecutive 252 patients were gastric cancer $(n=189,75 \%)$, adenoma $(n=26,10.3 \%)$, and gastritis $(n=37,14.7 \%)$.

\section{Risk factors for malignancy in atypical gland}

The mean age was $62.24 \pm 10.79$ years. The proportion of males was $74.6 \%$ in the group with gastric cancer, which was not significantly different among groups with different final diagnoses. The mean size of atypical gland in the gastric cancer group (20.4 mm) was larger than that of the adenoma group (8.8 $\mathrm{mm}$ ) or the gastritis group $(9.2 \mathrm{~mm}$ ) (both $\mathrm{p}<0.001$ by one-way analysis of variance). Depressed type and surface nodularity were significantly predominant in the group with gastric cancer than those in the group with adenoma or gastritis. Underlying mucosal atrophy/intestinal metaplasia and the status of $H$. pylo-
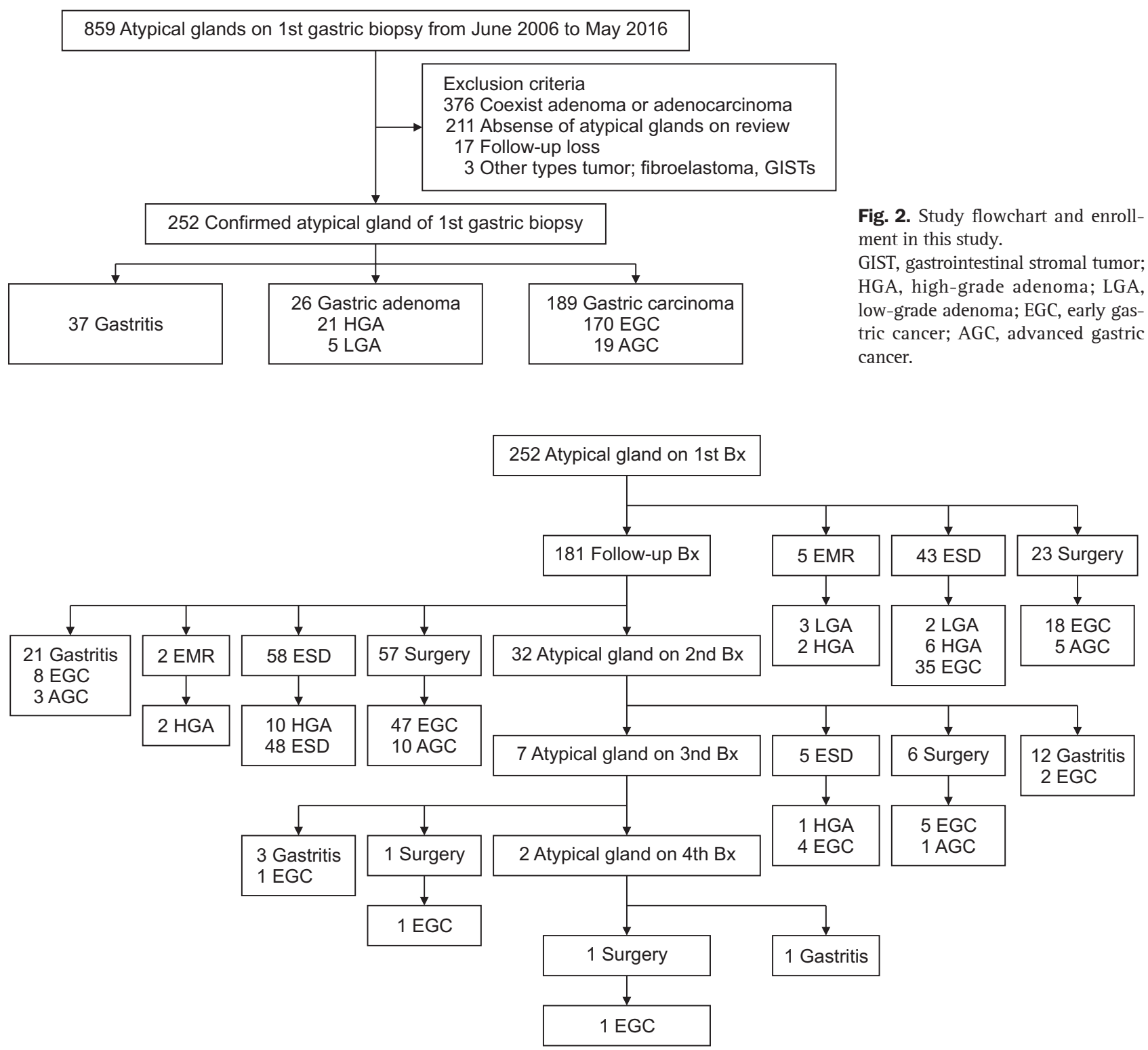

Fig. 3. Assessment and final diagnosis of atypical gland during initial biopsy.

Bx, biopsy; EMR, endoscopic mucosal resection; ESD, endoscopic submucosal dissection; LGA, low-grade adenoma; HGA, high-grade adenoma; EGC, early gastric cancer; AGC, advanced gastric cancer. 
ri infection were not significantly different among these groups (Table 1).

In addition, the malignant group and the benign group (including adenoma and gastritis) were compared and analyzed for clinical and endoscopic factors related to gastric cancer. The proportion of male was $74.6 \%$ in the malignant group and $77.8 \%$ in the benign group. The mean age of patients was 61.5 years in the malignant group and 64.6 years in the benign group. Differences in these were not statistically significant between the two groups.

Univariate analysis of clinicopathologic factors revealed that lesion size greater than $10 \mathrm{~mm}$, surface nodularity, and surface depression were significant risk factors for malignancy (Table 2). In multivariate analysis, lesion size more than $10 \mathrm{~mm}(\mathrm{p}=0.002$; odds ratio [OR], 3.021; 95\% confidence interval [CI], 1.480 to 6.165), depressed morphology ( $\mathrm{p}=0.001$; OR, 3.181 95\% CI, 1.579 to 6.406), and surface nodularity ( $\mathrm{p}=0.001$; OR, 3.432; 95\% CI, 1.667 to 7.064$)$ remained significant risk factors for malignancy (Table 3).

\section{DISCUSSION}

Endoscopic biopsy is usually performed initially for the diagnosis of suspicious gastric lesion during endoscopy. ${ }^{10,11}$ However, the diagnosis with forceps biopsy is not always definite. It is often inconsistent with the final pathology due to heterogeneity of the tissue itself as well as the sampling process affected by the location, depth, and the number of biopsies. ${ }^{14}$ In addition, biopsy specimens diagnosed as dysplasia/adenoma may be evaluated differently from pathological viewpoints. Atypical gland is a broad concept used in all cases where it is difficult to distinguish between benign reactive lesion and dysplasia or

Table 2. Risk Factors for Gastric Cancer with Atypical Glands

\begin{tabular}{|c|c|c|c|}
\hline & $\begin{array}{c}\text { Malignancy } \\
\quad(n=189)\end{array}$ & $\begin{array}{r}\text { Benign } \\
(n=63)\end{array}$ & p-value \\
\hline Age, yr & $61.5 \pm 11.0$ & $64.6 \pm 10.4$ & 0.055 \\
\hline Male sex & $141(74.6)$ & 49 (77.8) & 0.736 \\
\hline Lesion size (>10 mm) & $148(78.3)$ & $29(46.0)$ & $<0.001$ \\
\hline Gross type & & & $<0.001$ \\
\hline Elevated & $18(9.5)$ & 22 (34.9) & \\
\hline Flat & 37 (19.6) & $15(23.8)$ & \\
\hline Depressed & $134(70.9)$ & $26(41.3)$ & \\
\hline Surface nodularity & 145 (76.7) & $28(44.4)$ & $<0.001$ \\
\hline Surface redness & $114(60.3)$ & $33(52.4)$ & 0.303 \\
\hline $\begin{array}{l}\text { Location of stomach } \\
\text { anatomy }\end{array}$ & & & 0.111 \\
\hline Lower & 129 (68.3) & $51(81.0)$ & \\
\hline Middle & $26(13.8)$ & $7(11.1)$ & \\
\hline Upper & $34(18.0)$ & $5(7.9)$ & \\
\hline Ulcer & $45(23.8)$ & $14(22.2)$ & 0.865 \\
\hline Gastric atrophy & $142(75.1)$ & $40(63.5)$ & 0.104 \\
\hline Intestinal metaplasia & $106(56.1)$ & $36(57.1)$ & 1 \\
\hline $\begin{array}{l}\text { Helicobacter pylori } \\
\text { positive }\end{array}$ & $62(50.4)$ & $31(50.8)$ & 1 \\
\hline
\end{tabular}

Data are presented as mean \pm SD or number $(\%)$.

Table 1. Baseline Characteristics of Patients with Atypical Glands on the First Biopsy

\begin{tabular}{|c|c|c|c|c|}
\hline Characteristic & Adenocarcinoma $(\mathrm{n}=189)$ & Adenoma $(n=26)$ & Gastritis $(n=37)$ & p-value \\
\hline Age, yr & $62.2 \pm 10.8$ & $63.7 \pm 12.4$ & $67.8 \pm 5.5$ & 0.695 \\
\hline Male sex & $141(74.6)$ & $18(69.2)$ & $31(83.8)$ & 0.368 \\
\hline Lesion size, $\mathrm{mm}$ & $20.4 \pm 15.4$ & $8.8 \pm 4.8$ & $9.2 \pm 5.3$ & $<0.001$ \\
\hline Gross type & & & & $<0.001$ \\
\hline Elevated & $18(9.5)$ & $14(53.8)$ & $8(21.6)$ & \\
\hline Flat & $37(19.6)$ & $5(19.2)$ & $10(27.0)$ & \\
\hline Depressed & $134(70.9)$ & 7 (26.9) & $19(51.4)$ & \\
\hline Surface nodularity & $145(76.7)$ & $16(61.5)$ & $12(32.4)$ & $<0.001$ \\
\hline Surface redness & $114(60.3)$ & $12(46.2)$ & $21(56.8)$ & 0.381 \\
\hline Location of stomach anatomy & & & & 0.240 \\
\hline Lower & $129(68.3)$ & $21(80.8)$ & $30(81.1)$ & \\
\hline Middle & $26(13.8)$ & $4(15.4)$ & $3(8.1)$ & \\
\hline Upper & $34(18.0)$ & 1 (3.8) & 4 (10.8) & \\
\hline Ulcer & $45(23.8)$ & $3(11.5)$ & $11(29.7)$ & 0.236 \\
\hline Gastric atrophy & $142(75.1)$ & $16(61.5)$ & 24 (64.9) & 0.266 \\
\hline Intestinal metaplasia & $106(56.1)$ & $18(69.2)$ & 18 (48.6) & 1 \\
\hline Helicobacter pylori positive & $62(50.4)$ & $13(52.0)$ & $18(50.0)$ & 0.987 \\
\hline
\end{tabular}

Data are presented as mean \pm SD or number $(\%)$. 
carcinoma. ${ }^{10,20}$ For lesion with severe inflammation and regeneration of gland, it may be difficult to differentiate between benign and malignant lesion by cellular and glandular atypism, especially for small amount of tissue in forceps biopsy. ${ }^{18}$ In this study, 75\% of patients who were initially diagnosed as atypical gland in forceps biopsy was finally identified as gastric cancer. In a previous study, 21.8\% (26/119) of indefinite neoplasia (category 2) were also confirmed to be gastric cancer. ${ }^{7}$ Therefore, atypical gland can be a diagnosis of broad spectrum from benign inflammatory lesion to cancer.

Few studies have dealt with clinical significance of indefinite neoplasia (category 2), including atypical gland. ${ }^{3,7}$ Precise diagnosis and grading of dysplasia are important in determining the treatment strategy. Although several studies have recommended further evaluation such as big size re-biopsy for strongly suspi- cious lesion of malignancy, ${ }^{8,13,19}$ endoscopic resection can be a modality for definite diagnosis and treatment of atypical gland. However, ESD may be an over-treatment for all cases of atypical gland.

In previous studies about dysplasia, endoscopic findings with lesion size greater than $2 \mathrm{~cm},{ }^{7}$ presence of ulcer, depressed morphology, ${ }^{19}$ and hemorrhagic tendency ${ }^{13}$ have strongly suggested high-grade adenoma or gastric cancer. ${ }^{20}$ In revised Vienna classification category 2, lesion greater than $1 \mathrm{~cm}$ in diameter and surface discoloration were risk factors for carcinoma. ${ }^{7}$ However, previous studies have focused on general endoscopic findings of patients with dysplasia or inflammatory lesions such as regenerative atypism/atypia, instead of focusing on atypical glands only. In this study, only patients with atypical glands from initial gastric biopsy were included. Lesion size greater than $1 \mathrm{~cm}$, surface

Table 3. Significant Risk Factors for Gastric Cancer with Atypical Glands by Univariate Analysis \& Multivariate Analysis

\begin{tabular}{lccccc}
\hline \multirow{2}{*}{ Risk factors } & \multicolumn{2}{c}{ Univariate analysis } & \multicolumn{2}{c}{ Multivariate analysis } \\
\cline { 2 - 5 } \cline { 4 - 6 } & p-value & OR (95\% CI) & p-value & OR (95\% CI) \\
\hline Lesion size $(>10 \mathrm{~mm})$ & $<0.001$ & $4.23(2.31-7.74)$ & 0.002 & $3.02(1.48-6.17)$ \\
Mucosal depression & $<0.001$ & $3.06(1.68-5.55)$ & 0.001 & $3.18(1.58-6.41)$ \\
Surface nodularity & $<0.001$ & $4.12(2.26-7.51)$ & 0.001 & $3.43(1.67-7.06)$ \\
\hline
\end{tabular}

OR, odds ratio; CI, confidence interval.

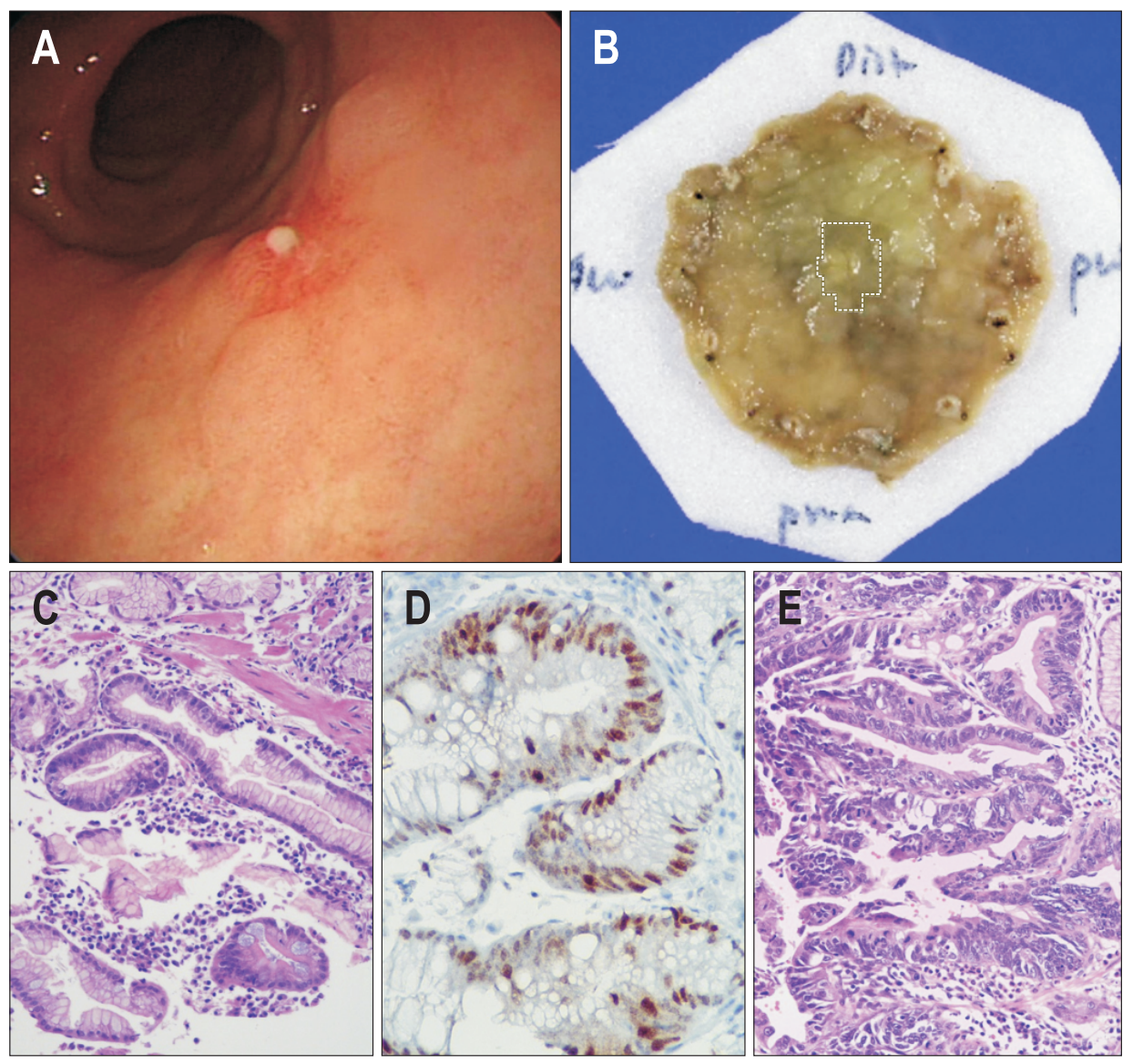

Fig. 4. A case of definite diagnosis after endoscopic submucosal dissection (ESD) in atypical glands during initial biopsy. (A) Hyperemic depressed mucosal nodularity on the antrum posterior wall. (B) Post-ESD histopathological specimen fixed with formalin (white dashed line on the borderline of an adenocarcinoma by pathologic mapping). (C) Histological finding of atypical glands during initial biopsy (H\&E, ×200). (D) Positive immunochemical staining during the initial biopsy (p53, x400). (E) Well-differentiated adenocarcinoma on post-ESD pathology (H\&E, $\times 200$ ). 
depression, and surface nodularity were found to be significant risk factors for malignancy. When only EGC and benign lesions were compared, lesion greater than $10 \mathrm{~mm}$ and surface nodularity were also risk factors for EGC (Supplementary Tables 1 and 2). Undifferentiated pathology is known to be a reasonable treatment for surgery. We also performed subgroup analysis for undifferentiated and differentiated carcinoma in gastric cancer. Lesions size of more than $2 \mathrm{~cm}$ and surface depression were also found to be significant risk factors for undifferentiated type (Supplementary Table 3).

In some cases of atypical glands on biopsy, immunohistochemical staining performed using p53 before ESD or surgery might be helpful for the prediction of malignancy. This stainingcan be a useful tool for the prediction of malignancy in cases with atypical glands (Fig. 4). ${ }^{21}$

This study had several limitations. The interpretation of atypical gland could be influenced by intra- and inter-observer variation. ${ }^{22-24}$ In this study, all slides were reviewed by two pathologists to minimize the inter-observer variation. Second, there could be a selection bias due to its retrospective nature. Third, only conventional endoscopic findings were included in the analysis without additional information such as narrowband imaging, ${ }^{25}$ chromoendoscopic imaging, ${ }^{26}$ or concomitant medication. ${ }^{27}$ Finally, we did not evaluate features of atypical gland that were suggestive of benign lesion. In the management of gastric atypical gland, it is important to exclude benign lesions that do not require an invasive diagnostic approach. Therefore, it is necessary to analyze predictors suggesting benign lesion. Further investigation is warranted.

In conclusion, atypical gland can contain malignancy, especially when there is large sized ( $>10 \mathrm{~mm}$ ) lesion, depressed morphology, or surface nodularity. Accurate diagnosis by rebiopsy or definitive treatment is mandatory by endoscopic or surgical resection in the suspicion of malignancy. Simple endoscopic follow-up with re-biopsy might be insufficient. It might delay the diagnosis and miss the appropriate treatment period. Repeated examinations can also lead to cost and psychologi$\mathrm{cal} /$ social stress. If the above suspicious malignant findings are present, aggressive methods can be applied simultaneously with diagnosis. Treatment such as ESD can be considered.

\section{CONFLICTS OF INTEREST}

No potential conflict of interest relevant to this article was reported.

\section{ACKNOWLEDGEMENTS}

This research was supported by a grant (NRF-2017R1D1A1B03036304) of the Basic Science Research Program through the National Research Foundation (NRF) funded by the Ministry of Education, Republic of Korea.

\section{REFERENCES}

1. Rahman R, Asombang AW, Ibdah JA. Characteristics of gastric cancer in Asia. World J Gastroenterol 2014;20:4483-4490.

2. Kim Y, Jun JK, Choi KS, Lee HY, Park EC. Overview of the National Cancer Screening Programme and the cancer screening status in Korea. Asian Pac J Cancer Prev 2011;12:725-730.

3. Min BH, Kang KJ, Lee JH, et al. Endoscopic resection for undifferentiated early gastric cancer: focusing on histologic discrepancies between forceps biopsy-based and endoscopic resection specimenbased diagnosis. Dig Dis Sci 2014;59:2536-2543.

4. Dinis-Ribeiro M, Areia M, de Vries AC, et al. Management of precancerous conditions and lesions in the stomach (MAPS): guideline from the European Society of Gastrointestinal Endoscopy (ESGE), European Helicobacter Study Group (EHSG), European Society of Pathology (ESP), and the Sociedade Portuguesa de Endoscopia Digestiva (SPED). Endoscopy 2012;44:74-94.

5. Hirota WK, Zuckerman MJ, Adler DG, et al. ASGE guideline: the role of endoscopy in the surveillance of premalignant conditions of the upper GI tract. Gastrointest Endosc 2006;63:570-580.

6. Lee JH, Min YW, Lee JH, et al. Diagnostic group classifications of gastric neoplasms by endoscopic resection criteria before and after treatment: real-world experience. Surg Endosc 2016;30:39873993.

7. Goo JJ, Choi CW, Kang DH, et al. Risk factors associated with diagnostic discrepancy of gastric indefinite neoplasia: who need en bloc resection? Surg Endosc 2015;29:3761-3767.

8. Cho SJ, Choi IJ, Kim CG, et al. Risk of high-grade dysplasia or carcinoma in gastric biopsy-proven low-grade dysplasia: an analysis using the Vienna classification. Endoscopy 2011;43:465-471.

9. Fletcher CD. Diagnostic histopathology of tumors. Philadelphia: Elsevier Health Sciences, 2013.

10. Dixon MF. Gastrointestinal epithelial neoplasia: Vienna revisited. Gut 2002;51:130-131.

11. Schlemper RJ, Riddell RH, Kato Y, et al. The Vienna classification of gastrointestinal epithelial neoplasia. Gut 2000;47:251-255.

12. Feldman M, Friedman LS, Brandt LJ. Sleisenger and Fordtran's gastrointestinal and liver disease: pathophysiology, diagnosis, management. Expert Consult Premium Edition-Enhanced Online Features. Philadelphia: Elsevier Health Sciences, 2010.

13. Ryu DG, Choi CW, Kang DH, et al. Clinical outcomes of endoscopic submucosa dissection for high-grade dysplasia from endoscopic forceps biopsy. Gastric Cancer 2017;20:671-678.

14. Kato M, Nishida T, Tsutsui S, et al. Endoscopic submucosal dissection as a treatment for gastric noninvasive neoplasia: a multicenter study by Osaka University ESD Study group. J Gastroenterol 2011;46:325-331.

15. The Paris endoscopic classification of superficial neoplastic lesions: esophagus, stomach, and colon: November 30 to December 1, 2002. Gastrointest Endosc 2003;58(6 Suppl):S3-S43.

16. Japanese Gastric Cancer Association. Japanese classification of gastric carcinoma: 3rd English edition. Gastric Cancer 2011;14: 
101-112.

17. Kleihues P, Cavenee W. World Health Organization classification of tumours: pathology and genetics of tumours of the nervous system. Lyon: IARC, 2000.

18. Brien TP, Farraye FA, Odze RD. Gastric dysplasia-like epithelial atypia associated with chemoradiotherapy for esophageal cancer: a clinicopathologic and immunohistochemical study of 15 cases. Mod Pathol 2001;14:389-396.

19. Choi CW, Kim HW, Shin DH, et al. The risk factors for discrepancy after endoscopic submucosal dissection of gastric category 3 lesion (low grade dysplasia). Dig Dis Sci 2014;59:421-427.

20. Park DI, Rhee PL, Kim JE, et al. Risk factors suggesting malignant transformation of gastric adenoma: univariate and multivariate analysis. Endoscopy 2001;33:501-506.

21. Niimi C, Goto H, Ohmiya N, et al. Usefulness of p53 and Ki67 immunohistochemical analysis for preoperative diagnosis of extremely well-differentiated gastric adenocarcinoma. Am J Clin Pathol 2002;118:683-692.

22. Flucke U, Mönig SP, Baldus SE, et al. Differences between biopsy- or specimen-related Laurén and World Health Organization classification in gastric cancer. World J Surg 2002;26:137-140.

23. Kim JM, Cho MY, Sohn JH, et al. Diagnosis of gastric epithelial neoplasia: dilemma for Korean pathologists. World J Gastroenterol 2011;17:2602-2610.

24. Shibata A, Longacre TA, Puligandla B, Parsonnet J, Habel LA. Histological classification of gastric adenocarcinoma for epidemiological research: concordance between pathologists. Cancer Epidemiol Biomarkers Prev 2001;10:75-78.

25. Nakayoshi T, Tajiri H, Matsuda K, Kaise M, Ikegami M, Sasaki H. Magnifying endoscopy combined with narrow band imaging system for early gastric cancer: correlation of vascular pattern with histopathology (including video). Endoscopy 2004;36:1080-1084.

26. Kono Y, Takenaka R, Kawahara Y, et al. Chromoendoscopy of gastric adenoma using an acetic acid indigocarmine mixture. World J Gastroenterol 2014;20:5092-5097.

27. Im JP, Kim SG, Kim JS, Jung HC, Song IS. Time-dependent morphologic change in depressed-type early gastric cancer. Surg Endosc 2009;23:2509-2514. 